\title{
Characterizations of Bloch-Type Spaces of Harmonic Mappings
}

\author{
Munirah Aljuaid ${ }^{1}$ and Flavia Colonna $\mathbb{D}^{2}$ \\ ${ }^{1}$ Department. of Mathematics, Northern Borders University, Arar 73222, Saudi Arabia \\ ${ }^{2}$ Department of Mathematical Sciences, George Mason University, Fairfax, VA 22030, USA
}

Correspondence should be addressed to Flavia Colonna; fcolonna@gmu.edu

Received 22 April 2019; Accepted 28 May 2019; Published 5 August 2019

Academic Editor: Ruhan Zhao

Copyright @ 2019 Munirah Aljuaid and Flavia Colonna. This is an open access article distributed under the Creative Commons Attribution License, which permits unrestricted use, distribution, and reproduction in any medium, provided the original work is properly cited.

\begin{abstract}
We study the Banach space $\mathscr{B}_{H}^{\alpha}(\alpha>0)$ of the harmonic mappings $h$ on the open unit disk $\mathbb{D}$ satisfying the condition $\sup _{z \in \mathbb{D}}(1-$ $\left.|z|^{2}\right)^{\alpha}\left(\left|h_{z}(z)\right|+\left|h_{\bar{z}}(z)\right|\right)<\infty$, where $h_{z}$ and $h_{\bar{z}}$ denote the first complex partial derivatives of $h$. We show that several properties that are valid for the space of analytic functions known as the $\alpha$-Bloch space extend to $\mathscr{B}_{H}^{\alpha}$. In particular, we prove that for $\alpha>0$ the mappings in $\mathscr{B}_{H}^{\alpha}$ can be characterized in terms of a Lipschitz condition relative to the metric defined by $d_{H, \alpha}(z, w)=\sup \{\mid h(z)-$ $\left.h(w) \mid: h \in \mathscr{B}_{H}^{\alpha},\|h\|_{\mathscr{B}_{H}^{\alpha}} \leq 1\right\}$. When $\alpha>1$, the harmonic $\alpha$-Bloch space can be viewed as the harmonic growth space of order $\alpha-1$, while for $0<\alpha<1, \mathscr{B}_{H}^{\alpha}$ is the space of harmonic mappings that are Lipschitz of order $1-\alpha$.
\end{abstract}

\section{Introduction}

Given a region $\Omega$ in the complex plane $\mathbb{C}$, a harmonic mapping with domain $\Omega$ is a complex-valued function $h$ defined on $\Omega$ satisfying the Laplace equation

$$
\Delta h:=4 h_{z \bar{z}} \equiv 0 \quad \text { on } \Omega \text {, }
$$

having denoted by $h_{z \bar{z}}$ the mixed complex second partial derivatives of $h$.

It is well known that a harmonic mapping $h$ admits a representation of the form $f+\bar{g}$, where $f$ and $g$ are analytic functions. This representation is unique if, fixing a base point $z_{0}$, the function $g$ is chosen so that $g\left(z_{0}\right)=0$.

In the last several decades, much research has been carried out on the study of Banach spaces of analytic functions on the open unit disk $\mathbb{D}$ in the complex plane. Since analytic functions are clearly harmonic, a natural question is whether such spaces $X$ are subspaces of some Banach space $X_{\mathscr{H}}$ of harmonic mappings on $\mathbb{D}$ in such a way that the norm on the larger space agrees with the norm of $X$ when restricting to the elements of $X$.

A space that has been thoroughly studied in complex function theory is the classical Bloch space $\mathscr{B}$ defined as the set of analytic functions $f$ on $\mathbb{D}$ such that

$$
\beta_{f}:=\sup _{z \in \mathbb{D}}\left(1-|z|^{2}\right)\left|f^{\prime}(z)\right|<\infty .
$$

In [1], Theorem 10, the second author observed that the functions $f$ in the Bloch space are precisely the analytic Lipschitz maps when regarded as functions between the metric spaces $(\mathbb{D}, \rho)$ (where $\rho$ denotes the hyperbolic distance) and $(\mathbb{C},|\cdot|)$ (see also Theorem 5.5 of [2]). Moreover, the correspondence $f \longmapsto \beta_{f}$ is a seminorm on $\mathscr{B}$ and is equal to the Lipschitz number, namely, for $f \in \mathscr{B}$,

$$
\beta_{f}=\sup _{z \neq w} \frac{|f(z)-f(w)|}{\rho(z, w)},
$$

where we recall

$$
\begin{aligned}
& \rho(z, w)=\frac{1}{2} \log \frac{1+\left|\phi_{z}(w)\right|}{1-\left|\phi_{z}(w)\right|}, \\
& \text { with } \phi_{z}(w)=\frac{z-w}{1-\bar{z} w} .
\end{aligned}
$$

This result motivated the following notion of Bloch harmonic mapping in [3].

A harmonic mapping $h$ on $\mathbb{D}$ is called Bloch if there exists a constant $c>0$ such that

$$
|h(z)-h(w)| \leq c \rho(z, w) \text { for all } z, w \in \mathbb{D}
$$


where for $h=f+\bar{g}$, with $f, g$ analytic on $\mathbb{D}$, the Lipschitz number

$$
L_{h}=\sup _{z \neq w} \frac{|h(z)-h(w)|}{\rho(z, w)}
$$

was shown in [3] to be equal to the quantity

$$
\begin{aligned}
\beta_{h} & :=\sup _{z \in \mathbb{D}}\left(1-|z|^{2}\right)\left(\left|f^{\prime}(z)\right|+\left|g^{\prime}(z)\right|\right) \\
& =\sup _{z \in \mathbb{D}}\left(1-|z|^{2}\right)\left(\left|h_{z}(z)\right|+\left|h_{\bar{z}}(z)\right|\right),
\end{aligned}
$$

thereby extending to harmonic mappings the corresponding result valid for analytic functions.

In this work, we expand the research done in [3] by focusing on the study of the harmonic extensions of the $\alpha$ Bloch spaces (for $\alpha>0$ ) introduced by Zhu in [4].

Research on the study of harmonic mappings has been conducted extensively in the last two decades by mostly analyzing the function theoretic aspects. The study of the extensions of classical Banach spaces of analytic functions is still relatively limited. Besides [3], references in the unit disk setting include [5] for the study of harmonic Bloch and Besov spaces, [6] for the study of harmonic $\nu$-Bloch mappings, [7] on planar harmonic Lipschitz and Hardy classes, and [8] for the study of harmonic Lipschitz-type spaces. In the setting of the unit ball in $\mathbb{C}^{n}$, see [9] for the study of the harmonic Bloch spaces, $[10,11]$ for the study of the harmonic Bergman spaces and [12] for extensions of the main results in [5]. For a general reference on harmonic mappings in the plane we refer the interested reader to [13].

After giving in Section 2 some preliminaries and background on the (analytic) $\alpha$-Bloch spaces, we introduce in Section 3 the harmonic $\alpha$-Bloch spaces and study their properties. In particular, we show that as done by the second author in [3] for the harmonic Bloch space (case $\alpha=1$ ), the $\alpha$-Bloch space can be characterized by Lipschitz-type conditions similar to the conditions obtained by Zhu in [4] for other positive values of $\alpha$.

In Section 4 , we show that for $\alpha \neq 1$, as for the analytic counterparts described by Zhu in [4], such spaces can be divided into two classes: the space of Lipschitz harmonic mappings of order $1-\alpha$ for $0<\alpha<1$, and the harmonic weighted Banach space of harmonic mappings with weight given by the Bergman weight $z \longmapsto\left(1-|z|^{2}\right)^{\alpha-1}$ for $\alpha>1$.

Finally, in Section 5, we give some properties of the harmonic growth spaces that are useful to extend a characterization of functions in the Zygmund space to the harmonic space counterpart. This topic is treated in [14].

\section{Preliminaries and Background}

Let $H(\mathbb{D})$ denote the class of analytic functions on $\mathbb{D}$, and for $R>0$, let $\mathbb{D}(0, R)$ be the open disk centered at 0 of radius $R$.

In [4], for $\alpha>0$, Zhu introduced the $\alpha$-Bloch space $\mathscr{B}^{\alpha}$ as the collection of functions $f \in H(\mathbb{D})$ such that

$$
\beta_{f}^{\alpha}:=\sup _{z \in \mathbb{D}}\left(1-|z|^{2}\right)^{\alpha}\left|f^{\prime}(z)\right|<\infty .
$$

The correspondence $f \longmapsto \beta_{f}^{\alpha}$ is a seminorm and $\mathscr{B}^{\alpha}$ is a Banach space under the norm

$$
\|f\|_{\mathscr{B}^{\alpha}}=|f(0)|+\beta_{f}^{\alpha} .
$$

For $\alpha=1, \mathscr{B}^{\alpha}$ is with the classical Bloch space $\mathscr{B}$. Thus the $\alpha$-Bloch space $\mathscr{B}^{\alpha}$ can be considered as the space of functions $f \in H(\mathbb{D})$ such that $f^{\prime}$ is in the growth space $\mathscr{A}^{-\alpha}$, defined as the collection of functions $g \in H(\mathbb{D})$ satisfying the growth condition

$$
|g(z)|=O\left((1-|z|)^{-\alpha}\right) .
$$

The subspace $\mathscr{B}_{0}^{\alpha}$ of $\mathscr{B}^{\alpha}$ consisting of the functions $f$ satisfying the condition

$$
\lim _{|z| \rightarrow 1}\left(1-|z|^{2}\right)^{\alpha}\left|f^{\prime}(z)\right|=0,
$$

known as the little $\alpha$-Bloch space, is the closure in $\mathscr{B}^{\alpha}$ of the polynomials and hence separable. Again, this subspace can be viewed as the collection of functions in $H(\mathbb{D})$ whose derivative is in the little growth space $\mathscr{A}_{0}^{-\alpha}$, whose members $f$ satisfy the "little oh" version of $(10)$ as $|z| \longrightarrow 1$.

Zhu proved that for a positive number $\alpha \neq 1$, the $\alpha$-Bloch space $\mathscr{B}^{\alpha}$ can be identified with one of two families of spaces, depending on whether $0<\alpha<1$ or $\alpha>1$. Specifically, (see Proposition 9 in [4]) if $0<\alpha<1$, then an analytic function $f$ on $\mathbb{D}$ belongs to $\mathscr{B}^{\alpha}$ if and only if

$$
\sup _{z \neq w} \frac{|f(z)-f(w)|}{|z-w|^{1-\alpha}}<\infty .
$$

In particular, the space $\mathscr{B}^{\alpha}$ is contained in the disk algebra.

In Proposition 7 of [4] it was shown that for $\alpha>$ 1 , the space $\mathscr{B}^{\alpha}$ (respectively, $\mathscr{B}_{0}^{\alpha}$ ) is the growth space $\mathscr{A}^{1-\alpha}$ (respectively, the little growth space $\mathscr{A}_{0}^{1-\alpha}$ ) and the corresponding norms are equivalent, where for $\gamma>0$ the norm of a function $f \in \mathscr{A}^{-\gamma}$ is defined as

$$
\|f\|_{\mathscr{A}^{-\gamma}}:=\sup _{z \in \mathbb{D}}\left(1-|z|^{2}\right)^{\gamma}|f(z)| .
$$

For more information on the growth spaces we refer the interested reader to [15].

The following result proved by Zhu in [4] shows that, for any integer $n \geq 2$, the elements of the $\alpha$-Bloch space can be characterized in terms of their $n^{\text {th }}$ derivative and a certain Bergman weight dependent of $n$ and $\alpha$.

Theorem 1 ([4], Proposition 8). Let $\alpha>0, n \geq 2$ be an integer, and $f \in H(\mathbb{D})$. Then

(i) $f \in \mathscr{B}^{\alpha}$ if and only if

$$
\beta_{f, n}^{\alpha}:=\sup _{z \in \mathbb{D}}\left(1-|z|^{2}\right)^{\alpha+n-1}\left|f^{(n)}(z)\right|<\infty .
$$

(ii) $f \in \mathscr{B}_{0}^{\alpha}$ if and only if

$$
\lim _{|z| \longrightarrow 1}\left(1-|z|^{2}\right)^{\alpha+n-1}\left|f^{(n)}(z)\right|=0 .
$$


Given $R>0$, we recall the Poisson integral representation of bounded real-valued harmonic functions on the disk $\mathbb{D}(0, R):=\{z \in \mathbb{C}:|z|<R\}$ with continuous boundary values (see, e.g., [16], (2.13), p. 260), extended in the obvious way to harmonic mappings.

Theorem 2. For $R>0$, a complex-valued continuous function $h$ on $\overline{\mathbb{D}(0, R)}$ and harmonic on $\mathbb{D}(0, R)$, admits the Poisson integral representation:

$$
h(z)=\frac{1}{2 \pi} \int_{-\pi}^{\pi} \frac{R^{2}-|z|^{2}}{\left|R e^{i t}-z\right|^{2}} h\left(R e^{i t}\right) d t
$$

for $|z|<R$.

This representation yields the following integral formulas for the complex partial derivatives of a harmonic mapping on $\mathbb{D}(0, R)$ with continuous extension to $\overline{\mathbb{D}(0, R)}$.

Theorem 3. Let $h$ be a complex-valued continuous function on $\overline{\mathbb{D}(0, R)}$ and harmonic on $\mathbb{D}(0, R)$. Then for $z \in \mathbb{D}(0, R)$,

$$
\begin{aligned}
& h_{z}(z)=\frac{1}{2 \pi} \int_{-\pi}^{\pi} \frac{R e^{i t}}{\left(R e^{i t}-z\right)^{2}} h\left(R e^{i t}\right) d t \\
& h_{\bar{z}}(z)=\frac{1}{2 \pi} \int_{-\pi}^{\pi} \frac{R e^{-i t}}{\left(R e^{-i t}-\bar{z}\right)^{2}} h\left(R e^{i t}\right) d t .
\end{aligned}
$$

Proof. Let us evaluate the partial derivatives with respect to $z$ and $\bar{z}$ of the Poisson kernel:

$$
\begin{aligned}
\frac{\partial}{\partial z} \frac{R^{2}-|z|^{2}}{\left|R e^{i t}-z\right|^{2}} & =\frac{R e^{i t}}{R e^{-i t}-\bar{z}} \frac{R e^{-i t}-\bar{z}}{\left(R e^{i t}-z\right)^{2}}=\frac{R e^{i t}}{\left(R e^{i t}-z\right)^{2}} \\
\frac{\partial}{\partial \bar{z}} \frac{R^{2}-|z|^{2}}{\left|R e^{i t}-z\right|^{2}} & =\frac{R e^{-i t}}{R e^{i t}-z} \frac{R e^{i t}-z}{\left(\operatorname{Re}^{-i t}-\bar{z}\right)^{2}} \\
& =\frac{R e^{-i t}}{\left(\operatorname{Re}^{-i t}-\bar{z}\right)^{2}}
\end{aligned}
$$

The results follow by applying (19) and (20) after differentiating (16) with respect to $z$ and $\bar{z}$ under the integral sign.

Remark 4. In the special case when the function $h$ is constant on the unit circle, the harmonic extension to $\mathbb{D}$ is constant as well, so its complex partial derivatives are identically 0 .

With the goal of characterizing functions in $\mathscr{B}^{\alpha}$ by a Lipschitz type condition, Zhu proved the following result.

Proposition 5 ([4], Proposition 16). For $\alpha>0$, and $z, w \in \mathbb{D}$, let

$$
d_{\alpha}(z, w)=\sup \left\{|f(z)-f(w)|: \beta_{f}^{\alpha} \leq 1\right\} .
$$

Then $d_{\alpha}$ defines a distance on $\mathbb{D}$.

In [4], Zhu observed that for $\alpha=1$, the metric $d_{\alpha}$ is precisely the hyperbolic metric $\rho$. To the best of our knowledge, an explicit formula of $d_{\alpha}$ for the case $\alpha \neq 1$ has not been determined.

The following result shows that the ratio of the distance $d_{\alpha}$ between two points in $\mathbb{D}$ to their Euclidean distance yields in the limit the reciprocal of the Bergman weight of order $\alpha$.

Theorem 6 ([4], Theorem 17). For any $\alpha>0$ and $z \in \mathbb{D}$,

$$
\lim _{w \longrightarrow z} \frac{d_{\alpha}(z, w)}{|z-w|}=\left(1-|z|^{2}\right)^{-\alpha} .
$$

This leads to the following Lipschitz-type characterization of analytic functions in $\mathscr{B}^{\alpha}$.

Theorem 7 ([4], Theorem 18). For $\alpha>0$ and $f \in H(\mathbb{D})$ the following statements are equivalent:

(1) $f \in \mathscr{B}^{\alpha}$.

(2) There exists a constant $c>0$ such that for all $z, w \in \mathbb{D}$

$$
|f(z)-f(w)| \leq c d_{\alpha}(z, w) .
$$

Moreover, for each $f \in \mathscr{B}^{\alpha}$,

$$
\beta_{f}^{\alpha}=\sup _{z \neq w} \frac{|f(z)-f(w)|}{d_{\alpha}(z, w)} .
$$

In the next section, after giving the notions of harmonic growth space and of harmonic $\alpha$-Bloch space, we follow a similar framework that will then allow us to extend Theorem 7 to harmonic mappings.

\section{Harmonic Growth Spaces and $\alpha$-Bloch Spaces}

For $\alpha>0$, we define the harmonic growth space $\mathscr{A}_{H}^{-\alpha}$ as the collection of all harmonic mappings $h$ on $\mathbb{D}$ such that

$$
\|h\|_{\mathscr{A}_{H}^{-\alpha}}:=\sup _{z \in \mathbb{D}}\left(1-|z|^{2}\right)^{\alpha}|h(z)|<\infty
$$

Theorem 8. The mapping $h \longmapsto\|h\|_{\mathscr{A}_{H}^{-\alpha}}$ defines a Banach space structure on $\mathscr{A}_{H}^{-\alpha}$ that extends the corresponding structure on $\mathscr{A}^{-\alpha}$.

Proof. It is straightforward to verify that $\|\cdot\|_{\mathscr{A}_{H}^{-\alpha}}$ is a norm. The space $\mathscr{A}^{-\alpha}$ is clearly a subspace of $\mathscr{A}_{H}^{-\alpha}$ and the respective norms coincide. To prove completeness, assume $\left(h_{n}\right)$ is a Cauchy sequence in $\mathscr{A}_{H}^{-\alpha}$. Fix $z \in \mathbb{D}$ and $\varepsilon>0$. Choose $N \in \mathbb{N}$ such that

$$
\left\|h_{n}-h_{m}\right\|_{\mathscr{A}_{H}^{-\alpha}}<\frac{\varepsilon}{\left(1-|z|^{2}\right)^{-\alpha}+1}
$$

for all $n, m \geq N$. Then for all $n, m \geq N$,

$$
\left|h_{n}(z)-h_{m}(z)\right| \leq\left(1-|z|^{2}\right)^{-\alpha}\left\|h_{n}-h_{m}\right\|_{\mathscr{A}_{H}^{-\alpha}}<\varepsilon .
$$

Thus, $\left(h_{n}(z)\right)$ is a Cauchy sequence in $\mathbb{C}$, which is complete, and we may define $h(z):=\lim _{n \rightarrow \infty} h_{n}(z)$. Then $h$ is 
continuous on $\mathbb{D}$ and since harmonic mappings satisfy the mean value property, for each $z \in \mathbb{D}$ and $r \in(0,1-|z|)$ and $n \in \mathbb{N}$,

$$
h_{n}(z)=\frac{1}{2 \pi} \int_{0}^{2 \pi} h_{n}\left(z+r e^{i t}\right) d t
$$

Passing to the limit as $n \longrightarrow \infty$, it follows that $h$ satisfies the mean value property. By Theorem 2.11 in [16] extended to complex-valued functions, $h$ is harmonic.

Since Cauchy sequences are bounded, $L:=\sup _{n \in \mathbb{N}}\left\|h_{n}\right\|_{\mathscr{A}_{H}^{-\alpha}}$ is finite. Fixing $z \in \mathbb{D}$, we have

$$
\left(1-|z|^{2}\right)^{\alpha}|h(z)|=\lim _{n \longrightarrow \infty}\left(1-|z|^{2}\right)^{\alpha}\left|h_{n}(z)\right| \leq L .
$$

Therefore, $h \in \mathscr{A}_{H}^{-\alpha}$ and $\|h\|_{\mathscr{A}_{H}^{-\alpha}} \leq L$.

To prove that $h_{n}$ converges to $h$ in norm, note that for $z \in$ $\mathbb{D}$, and $n, m \geq N$,

$$
\left(1-|z|^{2}\right)^{\alpha}\left|h_{n}(z)-h_{m}(z)\right| \leq\left\|h_{n}-h_{m}\right\|_{\mathscr{A}_{H}^{-\alpha}}<\varepsilon .
$$

Fixing $n \geq N$ and letting $m \longrightarrow \infty$, we get

$$
\left(1-|z|^{2}\right)^{\alpha}\left|h_{n}(z)-h(z)\right| \leq \varepsilon .
$$

Taking the supremum over all $z \in \mathbb{D}$, we obtain $\| h_{n}-$ $h \|_{\mathscr{A}_{H}^{-\alpha}} \longrightarrow 0$, as desired.

Define the little harmonic growth space $\mathscr{A}_{H, 0}^{-\alpha}$ as the subspace of $\mathscr{A}_{H}^{-\alpha}$ whose elements $h$ satisfy

$$
\lim _{|z| \longrightarrow 1}\left(1-|z|^{2}\right)^{\alpha}|h(z)|=0 .
$$

It is evident from the definition that if $f, g \in \mathscr{A}^{-\alpha}$ (respectively, $\mathscr{A}_{0}^{-\alpha}$ ), then $f+\bar{g} \in \mathscr{A}_{H}^{-\alpha}$ (respectively, $\mathscr{A}_{H, 0}^{-\alpha}$ ). We shall prove that the converse holds as well.

For $\alpha>0$, we now define the harmonic $\alpha$-Bloch space $\mathscr{B}_{H}^{\alpha}$ and in Section 4, we shall prove that in analogy to the analytic case, for $\alpha>1$, as sets, $\mathscr{B}_{H}^{\alpha}$ and the harmonic growth space $\mathscr{A}_{H}^{-(\alpha-1)}$ are equal, whereas for $0<\alpha<1$, the space $\mathscr{B}_{H}^{\alpha}$ is the Lipschitz space of harmonic mappings of order $1-\alpha$.

Definition 9. For $\alpha>0$, we define the harmonic $\alpha$-Bloch space $\mathscr{B}_{H}^{\alpha}$ as the collection of all harmonic mappings $h$ on $\mathbb{D}$ such that

$$
\beta_{h}^{\alpha}:=\sup _{z \in \mathbb{D}}\left(1-|z|^{2}\right)^{\alpha}\left(\left|h_{z}(z)\right|+\left|h_{\bar{z}}(z)\right|\right)<\infty .
$$

Clearly, when $\alpha=1$ this definition agrees with expression (7).

The harmonic little $\alpha$-Bloch space is defined as the subspace $\mathscr{B}_{H, 0}^{\alpha}$ of $\mathscr{B}_{H}^{\alpha}$ consisting of the mappings $h$ such that

$$
\lim _{|z| \longrightarrow 1}\left(1-|z|^{2}\right)^{\alpha}\left(\left|h_{z}(z)\right|+\left|h_{\bar{z}}(z)\right|\right)=0 .
$$

Observe that if $h=f+\bar{g}$ with $f, g \in H(\mathbb{D})$, then $h_{z}=f^{\prime}$ and $h_{\bar{z}}=\overline{g^{\prime}}$. Thus

$$
\beta_{h}^{\alpha}=\sup _{z \in \mathbb{D}}\left(1-|z|^{2}\right)^{\alpha}\left(\left|f^{\prime}(z)\right|+\left|g^{\prime}(z)\right|\right) .
$$

This implies that

$$
\frac{1}{2}\left(\beta_{f}^{\alpha}+\beta_{g}^{\alpha}\right) \leq \max \left\{\beta_{f}^{\alpha}, \beta_{g}^{\alpha}\right\} \leq \beta_{h}^{\alpha} \leq \beta_{f}^{\alpha}+\beta_{g}^{\alpha} .
$$

We deduce the following result.

Proposition 10. Let $\alpha>0$ and let $h$ be a harmonic mapping, $h=f+\bar{g}$ with $f, g \in H(\mathbb{D})$. Then $h \in \mathscr{B}_{H}^{\alpha}$ (respectively, $h \in \mathscr{B}_{H, 0}^{\alpha}$ ) if and only if $f$ and $g$ are in $\mathscr{B}^{\alpha}$ (respectively, $f$ and $g$ are in $\left.\mathscr{B}_{0}^{\alpha}\right)$.

In particular, since for $0<\alpha<1$, the space $\mathscr{B}^{\alpha}$ is contained in the disk algebra, the space $\mathscr{B}_{H}^{\alpha}$ is contained in the space of complex-valued harmonic functions in $\mathbb{D}$ which are continuous on $\overline{\mathbb{D}}$.

It is straightforward to verify that the mapping

$$
h \longmapsto\|h\|_{\mathscr{B}_{H}^{\alpha}}:=|h(0)|+\beta_{h}^{\alpha}
$$

defines a Banach space structure on $\mathscr{B}_{H}^{\alpha}$, which extends to harmonic mappings the norm on $\mathscr{B}^{\alpha}$.

A natural question that arises is whether Theorems 6 and 7 extend to harmonic mappings. We shall show that this is indeed the case. We make use of the following result whose proof is elementary.

Proposition 11. For $\alpha>0$, the function $d_{H, \alpha}$ defined by

$$
\begin{aligned}
& d_{H, \alpha}(z, w) \\
& =\sup \left\{|h(z)-h(w)|: h \in \mathscr{B}_{H}^{\alpha}, \quad \beta_{h}^{\alpha} \leq 1\right\}, \\
& \qquad \text { for } z, w \in \mathbb{D},
\end{aligned}
$$

is a distance on $\mathbb{D}$.

Theorem 12. For any $\alpha>0$ and $z \in \mathbb{D}$,

$$
\lim _{w \longrightarrow z} \frac{d_{H, \alpha}(z, w)}{|z-w|}=\left(1-|z|^{2}\right)^{-\alpha} .
$$

Proof. By Theorem 17 of [4], for $z \in \mathbb{D}$,

$$
\lim _{w \longrightarrow z} \frac{d_{\alpha}(z, w)}{|z-w|}=\left(1-|z|^{2}\right)^{-\alpha} .
$$

From the definitions of $d_{H, \alpha}$ and $d_{\alpha}$, it is immediate to see that $d_{H, \alpha}(z, w) \geq d_{\alpha}(z, w)$, for all $z, w \in \mathbb{D}$. Hence, fixing $z \in \mathbb{D}$, we have

$$
\liminf _{w \longrightarrow z} \frac{d_{H, \alpha}(z, w)}{|z-w|} \geq \lim _{w \longrightarrow z} \frac{d_{\alpha}(z, w)}{|z-w|}=\left(1-|z|^{2}\right)^{-\alpha} .
$$

Therefore, to prove the result, it suffices to show that for each $z \in \mathbb{D}$

$$
\limsup _{w \rightarrow z} \frac{d_{H, \alpha}(z, w)}{|z-w|} \leq\left(1-|z|^{2}\right)^{-\alpha}
$$

Fix $z \in \mathbb{D}$ and let $h \in \mathscr{B}_{H}^{\alpha}$ with $\beta_{h}^{\alpha} \leq 1$. Replacing $h$ with $h-$ $h(0)$, we may assume $h(0)=0$, so that $\|h\|_{\mathscr{B}_{H}^{\alpha}}=\beta_{h}^{\alpha}$. Represent 
$h$ as $f+\bar{g}$ with $f, g$ analytic and $f(0)=g(0)=0$. Proceeding as in the proof of Theorem 17 of [4], fix $z \in \mathbb{D}$ and let $r=$ $(1-|z|) / 2$. Then for $|w-z|<r$,

$$
\begin{aligned}
h(w)= & h(z)+f^{\prime}(z)(w-z)+\overline{g^{\prime}(z)}(\bar{w}-\bar{z}) \\
& +f_{2}(z)(w-z)^{2}+\overline{g_{2}(z)}(\bar{w}-\bar{z})^{2},
\end{aligned}
$$

where

$$
\begin{aligned}
f_{2}(w) & =\frac{1}{2 \pi i} \int_{|\zeta-z|=r} \frac{f(\zeta)}{(\zeta-z)^{2}(\zeta-w)} d \zeta \\
\text { and } g_{2}(w) & =\frac{1}{2 \pi i} \int_{|\zeta-z|=r} \frac{g(\zeta)}{(\zeta-z)^{2}(\zeta-w)} d \zeta
\end{aligned}
$$

By Proposition 5 of [4], there exists some positive constant $C$ only dependent on $z$ such that

$$
\begin{aligned}
& \left|f_{2}(w)\right| \leq C\|f\|_{\mathscr{B}^{\alpha}} \\
& \text { and }\left|g_{2}(w)\right| \leq C\|g\|_{\mathscr{B}^{\alpha}}
\end{aligned}
$$

for $|w-z|<r$. Therefore, since the assumption $\beta_{h}^{\alpha} \leq 1$ implies that

$$
\left|f^{\prime}(z)\right|+\left|g^{\prime}(z)\right| \leq\left(1-|z|^{2}\right)^{-\alpha},
$$

and noting that by (36), $\|f\|_{\mathscr{B}^{\alpha}}+\|g\|_{\mathscr{B}^{\alpha}} \leq 2\|h\|_{\mathscr{B}_{H}^{\alpha}} \leq 2$, using (46) we obtain

$$
\begin{aligned}
|h(z)-h(w)| \leq & |z-w|\left(\left|f^{\prime}(z)\right|+\left|g^{\prime}(z)\right|\right) \\
& +C|z-w|^{2}\left(\|f\|_{\mathscr{B}^{\alpha}}+\|g\|_{\mathscr{B}^{\alpha}}\right) \\
\leq & |z-w|\left(1-|z|^{2}\right)^{-\alpha}+2 C|z-w|^{2} .
\end{aligned}
$$

Hence,

$$
\frac{|h(z)-h(w)|}{|z-w|} \leq\left(1-|z|^{2}\right)^{-\alpha}+2 C|z-w| .
$$

Taking the supremum over all such mappings $h$, we obtain

$$
\frac{d_{H, \alpha}(z, w)}{|z-w|} \leq\left(1-|z|^{2}\right)^{-\alpha}+2 C|z-w|
$$

for $|w-z|<r$. Letting $w \longrightarrow z$, we obtain

$$
\limsup _{w \rightarrow z} \frac{d_{H, \alpha}(z, w)}{|z-w|} \leq\left(1-|z|^{2}\right)^{-\alpha},
$$

as desired.

We can now prove one of our main results in this section.

Theorem 13. Let $h$ be a harmonic mapping on $\mathbb{D}$ and $\alpha>0$. Then $h \in \mathscr{B}_{H}^{\alpha}$ if and only if there exists a constant $C>0$ such that for all $z, w \in \mathbb{D}$

$$
|h(z)-h(w)| \leq C d_{H, \alpha}(z, w) .
$$

Moreover, for each $h \in \mathscr{B}_{H}^{\alpha}$,

$$
\beta_{h}^{\alpha}=\sup _{z \neq w} \frac{|h(z)-h(w)|}{d_{H, \alpha}(z, w)} .
$$

Proof. Assume $h \in \mathscr{B}_{H}^{\alpha}$ and let $M_{h}^{\alpha}$ denote the right-hand side of (52). If $h$ is constant, then $M_{h}^{\alpha}=0=\beta_{h}^{\alpha}$, and we are done. So assume $h$ is nonconstant so that $\beta_{h}^{\alpha}>0$. Then, the mapping defined by

$$
\widetilde{h}=\frac{1}{\beta_{h}^{\alpha}} h
$$

is in $\mathscr{B}_{H}^{\alpha}$ and has seminorm 1 , so, fixing distinct points $z, w \in$ $\mathbb{D}$, by the definition of $d_{H, \alpha}$, we have

$$
|h(z)-h(w)|=\beta_{h}^{\alpha}|\widetilde{h}(z)-\tilde{h}(w)| \leq \beta_{h}^{\alpha} d_{H, \alpha}(z, w) .
$$

Dividing by $d_{H, \alpha}(z, w)$ and taking the supremum over all distinct points $z$ and $w$, we obtain $M_{h}^{\alpha} \leq \beta_{h}^{\alpha}$.

Next, note that fixing $z \in \mathbb{D}$,

$$
\begin{aligned}
M_{h}^{\alpha} & \geq \limsup _{w \longrightarrow z} \frac{|h(z)-h(w)|}{d_{H, \alpha}(z, w)} \\
& =\limsup _{w \longrightarrow z} \frac{|h(z)-h(w)|}{|z-w|} \lim _{w \longrightarrow z} \frac{|z-w|}{d_{H, \alpha}(z, w)} .
\end{aligned}
$$

For $w=z+r e^{i \theta}$ with $0<r<1-|z|$ and $\theta \in \mathbb{R}$ fixed,

$$
\begin{aligned}
& \frac{|h(w)-h(z)|}{|w-z|} \\
& =\left|\frac{f(w)-f(z)}{w-z}+\frac{\overline{g(w)}-\overline{g(z)}}{\bar{w}-\bar{z}} e^{-2 i \theta}\right| \\
& \longrightarrow\left|f^{\prime}(z)+\overline{g^{\prime}(z)} e^{-2 i \theta}\right|
\end{aligned}
$$

as $r \longrightarrow 0$. Taking the supremum over $\theta$, we obtain

$$
\limsup _{w \rightarrow z} \frac{|h(z)-h(w)|}{|z-w|}=\left|f^{\prime}(z)\right|+\left|g^{\prime}(z)\right| \text {. }
$$

Hence, by Theorem 12, from (55) we obtain

$$
M_{h}^{\alpha} \geq\left(\left|f^{\prime}(z)\right|+\left|g^{\prime}(z)\right|\right)\left(1-|z|^{2}\right)^{\alpha} .
$$

Taking the supremum over all $z \in \mathbb{D}$, we conclude that $M_{h}^{\alpha} \geq$ $\beta_{h}^{\alpha}$, completing the proof.

In [17], the norm of the point-evaluation functional on the Bloch space was calculated precisely. Specifically, it was shown that for $z \in \mathbb{D}$, the quantity

$$
\sup _{f \in \mathscr{B},\|f\|_{\mathscr{B}} \leq 1}|f(z)|=\max \{1, \rho(z, 0)\},
$$

where we recall that $\rho$ is the distance $d_{\alpha}$ for $\alpha=1$.

We now provide a point-evaluation estimate for the harmonic $\alpha$-Bloch space in terms of the metric $d_{H, \alpha}$ valid for all $\alpha>0$. For the case $\alpha=1$ we obtain an extension of (59) to the harmonic Bloch space $\mathscr{B}_{H}$.

Theorem 14. For $h \in \mathscr{B}_{H}^{\alpha}$ and $z \in \mathbb{D}$,

$$
|h(z)| \leq \max \left\{1, d_{H, \alpha}(z, 0)\right\}\|h\|_{\mathscr{B}_{H}^{\alpha}} .
$$

In the case when $\alpha=1$, for all $z \in \mathbb{D}$,

$$
\sup _{h \in \mathscr{B}_{H},\|h\|_{\mathscr{B}_{H}} \leq 1}|h(z)|=\max \{1, \rho(z, 0)\} .
$$


Proof. Let $h \in \mathscr{B}_{H}^{\alpha}$ and fix $z \in \mathbb{D}$. The inequality is clear for $z=0$. So assume $z \neq 0$. Then, by (52), we have

$$
\begin{aligned}
|h(z)| & \leq|h(0)|+|h(z)-h(0)| \\
& =|h(0)|+\frac{|h(z)-h(0)|}{d_{H, \alpha}(z, 0)} d_{H, \alpha}(z, 0) \\
& \leq|h(0)|+\beta_{h}^{\alpha} d_{H, \alpha}(z, 0) \\
& =|h(0)|+\left(\|h\|_{\mathscr{B}_{H}^{\alpha}}-|h(0)|\right) d_{H, \alpha}(z, 0) \\
& =\left(1-d_{H, \alpha}(z, 0)\right)|h(0)|+d_{H, \alpha}(z, 0)\|h\|_{\mathscr{B}_{H}^{\alpha}} \\
& =\max \left\{1, d_{H, \alpha}(z, 0)\right\}\|h\|_{\mathscr{B}_{H}^{\alpha}}
\end{aligned}
$$

proving the estimate.

When $\alpha=1$, by (59), we have

$$
\begin{aligned}
\max \{1, \rho(z, 0)\} & =\sup _{f \in \mathscr{B},\|f\|_{\mathscr{B}^{\alpha} \leq 1}}|f(z)| \\
& \leq \sup _{h \in \mathscr{B}_{H},\|h\|_{\mathscr{S}_{H}^{\alpha}} \leq 1}|h(z)|,
\end{aligned}
$$

which, combined with (62), yields the conclusion.

We now prove that for each $\alpha>0$, the map $h \longrightarrow \beta_{h}^{\alpha}$ is lower semicontinuous on the space $\mathscr{B}_{H}^{\alpha}$.

Theorem 15. Let $\left(h_{n}\right)$ be a sequence in $\mathscr{B}_{H}^{\alpha}$ converging uniformly on compact subsets of $\mathbb{D}$ to some function $h$. If the sequence $\left(\beta_{h_{n}}^{\alpha}\right)$ is bounded, then $h \in \mathscr{B}_{H}^{\alpha}$ and

$$
\beta_{h}^{\alpha} \leq \liminf _{n \longrightarrow \infty} \beta_{h_{n}}^{\alpha}
$$

Proof. Let $\left(h_{n}\right)$ be as in the statement of the theorem. Applying the mean value property of harmonic mappings to each $h_{n}$ and then passing to the limit as $n \longrightarrow \infty$, we see that $h$ also satisfies the mean value property and hence it is likewise harmonic. Set

$$
\beta=\liminf _{n \rightarrow \infty} \beta_{h_{n}}^{\alpha}
$$

Then, there exists a subsequence $\left(\beta_{h_{n_{k}}}^{\alpha}\right)$ converging to $\beta$. We wish to show that for all $z, w \in \mathbb{D}$,

$$
|h(z)-h(w)| \leq \beta d_{H, \alpha}(z, w) .
$$

Fix $z, w \in \mathbb{D}$. Since the above inequality is trivial if $z=w$, assume $z \neq w$ and fix $\varepsilon>0$. Since $\beta_{h_{n_{k}}}^{\alpha} \longrightarrow \beta$, there exists $\nu \in \mathbb{N}$ such that for all $k \geq v$

$$
\begin{aligned}
\beta_{h_{n_{k}}}^{\alpha} & <\beta+\frac{\varepsilon}{2 d_{H, \alpha}(z, w)}, \\
\left|h_{n_{k}}(z)-h(z)\right| & <\frac{\varepsilon}{4}, \\
\text { and }\left|h_{n_{k}}(w)-h(w)\right| & <\frac{\varepsilon}{4} .
\end{aligned}
$$

Therefore, for $k \geq v$, and having shown in Theorem 13 that the seminorm in $\mathscr{B}_{H}^{\alpha}$ equals the Lipschitz number with respect to the distance $d_{H, \alpha}$ and the Euclidean distance in $\mathbb{C}$, we have

$$
\begin{aligned}
|h(z)-h(w)| \leq & \left|h(z)-h_{n_{k}}(z)\right|+\left|h_{n_{k}}(z)-h_{n_{k}}(w)\right| \\
& +\left|h_{n_{k}}(w)-h(w)\right| \\
< & \frac{\varepsilon}{2}+\beta_{h_{n_{k}}}^{\alpha} d_{H, \alpha}(z, w) \\
< & \frac{\varepsilon}{2}+\left(\beta+\frac{\varepsilon}{2 d_{H, \alpha}(z, w)}\right) d_{H, \alpha}(z, w) \\
= & \varepsilon+\beta d_{H, \alpha}(z, w) .
\end{aligned}
$$

Since $\varepsilon$ is arbitrary, we conclude that $|h(z)-h(w)| \leq$ $\beta d_{H, \alpha}(z, w)$ for all $z, w \in \mathbb{D}$.

This implies that the Lipschitz number of $h$ is no greater than $\beta$. In particular, $h \in \mathscr{B}_{H}^{\alpha}$ and $\beta_{h}^{\alpha} \leq \beta$, completing the proof.

\section{Characterizations of the $\alpha$-Bloch Harmonic Mappings $(\alpha \neq 1)$}

We now introduce the space of harmonic Lipschitz mappings and prove that it is a complex Banach space.

For $\alpha>0$, let $\operatorname{Lip}_{H}^{\alpha}$ denote the collection of harmonic mappings $h$ on $\mathbb{D}$ satisfying the condition

$$
\|h\|_{\alpha}:=\sup _{z \neq w} \frac{|h(z)-h(w)|}{|z-w|^{\alpha}}<\infty .
$$

Define $\|h\|_{\operatorname{Lip}_{H}^{\alpha}}:=|h(0)|+\|h\|_{\alpha}$.

Theorem 16. For $\alpha>0$, under the above norm, $\operatorname{Lip}_{H}^{\alpha}$ is a Banach space.

Proof. It is immediate to verify that $\operatorname{Lip}_{H}^{\alpha}$ is a normed linear space. To prove completeness, suppose $\left(h_{n}\right)$ is a Cauchy sequence in $\operatorname{Lip}_{H}^{\alpha}$. Fix $z \in \mathbb{D}$ and $\varepsilon>0$. Choose $N \in \mathbb{N}$ such that

$$
\left\|h_{n}-h_{m}\right\|_{\operatorname{Lip}_{H}^{\alpha}}<\frac{\varepsilon}{|z|^{\alpha}+1}
$$

for all $n, m \geq N$. Then for all $n, m \geq N$,

$$
\begin{aligned}
\left|h_{n}(z)-h_{m}(z)\right| \leq & \left|h_{n}(z)-h_{m}(z)-h_{n}(0)-h_{m}(0)\right| \\
& +\left|h_{n}(0)-h_{m}(0)\right| \\
\leq & \left\|h_{n}-h_{m}\right\|_{\alpha}|z|^{\alpha}+\left|h_{n}(0)-h_{m}(0)\right| \\
\leq & \left(|z|^{\alpha}+1\right)\left\|h_{n}-h_{m}\right\|_{\operatorname{Lip}_{H}^{\alpha}<\varepsilon .}
\end{aligned}
$$

Thus, $\left(h_{n}(z)\right)$ is a Cauchy sequence in $\mathbb{C}$, which is complete, and so $h(z):=\lim _{n \rightarrow \infty} h_{n}(z)$ exists. Arguing as in the proof of Theorem 8 , we see that $h$ is harmonic.

Since Cauchy sequences are bounded,

$$
L:=\sup _{k \in \mathbb{N}}\left\|h_{k}\right\|_{\alpha} \leq \sup _{k \in \mathbb{N}}\left\|h_{k}\right\|_{\operatorname{Lip}_{H}^{\alpha}}<\infty .
$$


Then for $z \neq w$ fixed in $\mathbb{D}$, we have

$$
\frac{|h(z)-h(w)|}{|z-w|^{\alpha}}=\lim _{n \longrightarrow \infty} \frac{\left|h_{n}(z)-h_{n}(w)\right|}{|z-w|^{\alpha}} \leq L .
$$

Taking the supremum over all pairs of distinct points $z, w \in$ $\mathbb{D}$, we obtain $h \in \operatorname{Lip}_{H}^{\alpha}$ and $\|h\|_{\operatorname{Lip}_{H}^{\alpha}} \leq L$.

Lastly, to show that $\left\|h_{n}-h\right\|_{\operatorname{Lip}_{H}^{\alpha}} \longrightarrow 0$, note that with $z \in \mathbb{D}$ fixed, for $w \in \mathbb{D}$, with $w \neq z$, and $n, m \geq N$,

$$
\begin{aligned}
& \left|h_{n}(0)-h_{m}(0)\right| \\
& \quad+\frac{\left|h_{n}(z)-h_{m}(z)-\left(h_{n}(w)-h_{m}(w)\right)\right|}{|z-w|^{\alpha}}<\varepsilon .
\end{aligned}
$$

Letting $m \longrightarrow \infty$, we get

$$
\begin{aligned}
& \left|h_{n}(0)-h(0)\right|+\frac{\left|h_{n}(z)-h(z)-\left(h_{n}(w)-h(w)\right)\right|}{|z-w|^{\alpha}} \\
& \quad \leq \varepsilon,
\end{aligned}
$$

whence $\left\|h_{n}-h\right\|_{\operatorname{Lip}_{H}^{\alpha}} \longrightarrow 0$.

Theorem 17. For $0<\alpha<1$, as sets, $\mathscr{B}_{H}^{\alpha}=\operatorname{Lip}_{H}^{1-\alpha}$, and the respective norms are equivalent.

Proof. First, assume $h \in \mathscr{B}_{H}^{\alpha}$ and let $f, g \in H(\mathbb{D})$ such that $h=f+\bar{g}$ with $g(0)=0$. Then $f, g \in \mathscr{B}^{\alpha}$, so by Proposition 9 in [4], $f$ and $g$ belong to the Lipschitz space of analytic functions of order $1-\alpha$. Thus, for $z \neq w$,

$$
\begin{aligned}
\frac{|h(z)-h(w)|}{|z-w|^{1-\alpha}} & \leq \frac{|f(z)-f(w)|}{|z-w|^{1-\alpha}}+\frac{|g(z)-g(w)|}{|z-w|^{1-\alpha}} \\
& \leq\|f\|_{1-\alpha}+\|g\|_{1-\alpha} .
\end{aligned}
$$

Taking the supremum over all pairs of distinct points $z, w \in$ $\mathbb{D}$, we obtain

$$
\|h\|_{1-\alpha} \leq\|f\|_{1-\alpha}+\|g\|_{1-\alpha} .
$$

Hence $h \in \operatorname{Lip}_{H}^{1-\alpha}$. Since the space $\mathscr{B}^{\alpha}$ and the space $\operatorname{Lip}^{1-\alpha}$ of analytic functions on $\mathbb{D}$ which are Lipschitz of order $1-\alpha$ have equivalent norms, where for $f \in \operatorname{Lip}^{1-\alpha}$,

$$
\|f\|_{\operatorname{Lip}^{1-\alpha}}=|f(0)|+\|f\|_{1-\alpha},
$$

using the equalities $h(0)=f(0)$ and $g(0)=0$ and (36), we deduce that

$$
\begin{aligned}
\|h\|_{\operatorname{Lip}_{H}^{1-\alpha}} & =|h(0)|+\|h\|_{1-\alpha} \\
& \leq|f(0)|+\|f\|_{1-\alpha}+\|g\|_{1-\alpha} \\
& =\|f\|_{\operatorname{Lip}^{1-\alpha}}+\|g\|_{\operatorname{Lip}^{1-\alpha}} \leq C\left(\|f\|_{\mathscr{B}^{\alpha}}+\|g\|_{\mathscr{B}^{\alpha}}\right) \\
& \leq 2 C\left(|f(0)|+\max \left\{\beta_{f}^{\alpha}, \beta_{g}^{\alpha}\right\}\right) \\
& \leq 2 C\left(|h(0)|+\beta_{h}^{\alpha}\right)=2 C\|h\|_{\mathscr{B}_{H}^{\alpha}}
\end{aligned}
$$

for some positive constant $C$.
Conversely, assume $\|h\|_{1-\alpha}<\infty$. Fix $z=|z| e^{i \tau} \in \mathbb{D}$. By Theorem 3, making a change of variable and using Remark 4, and since $\left|e^{i(t+\tau)}-z\right|^{2}=\left|e^{i t}-\right| z||^{2}=\left|e^{-i(t+\tau)}-\bar{z}\right|^{2}$, we have

$$
\begin{aligned}
& \left|h_{z}(z)\right|+\left|h_{\bar{z}}(z)\right|=\left|\frac{1}{2 \pi} \int_{0}^{2 \pi} \frac{e^{i t}}{\left(e^{i t}-z\right)^{2}} h\left(e^{i t}\right) d t\right| \\
& +\left|\frac{1}{2 \pi} \int_{0}^{2 \pi} \frac{e^{-i t}}{\left(e^{-i t}-\bar{z}\right)^{2}} h\left(e^{i t}\right) d t\right| \\
& =\left|\frac{1}{2 \pi} \int_{0}^{2 \pi} \frac{e^{i t}}{\left(e^{i t}-z\right)^{2}}\left(h\left(e^{i t}\right)-h\left(e^{i \tau}\right)\right) d t\right| \\
& +\left|\frac{1}{2 \pi} \int_{0}^{2 \pi} \frac{e^{-i t}}{\left(e^{-i t}-\bar{z}\right)^{2}}\left(h\left(e^{i t}\right)-h\left(e^{i \tau}\right)\right) d t\right| \\
& \leq \frac{1}{2 \pi} \int_{-\pi}^{\pi} \frac{\left|h\left(e^{i(t+\tau)}\right)-h\left(e^{i \tau}\right)\right|}{\left|e^{i(t+\tau)}-z\right|^{2}} d t \\
& +\frac{1}{2 \pi} \int_{-\pi}^{\pi} \frac{\left|h\left(e^{i(t+\tau)}\right)-h\left(e^{i \tau}\right)\right|}{\left|e^{-i(t+\tau)}-\bar{z}\right|^{2}} d t . \\
& =\frac{1}{\pi} \int_{-\pi}^{\pi} \frac{\left|h\left(e^{i(t+\tau)}\right)-h\left(e^{i \tau}\right)\right|}{\left|e^{i t}-\right| z||^{2}} d t .
\end{aligned}
$$

By the definition of $\|h\|_{1-\alpha}$, and expanding $e^{i t}$ as a power series in $t$ about 0 , for $|t| \leq \pi$, we have

$$
\begin{aligned}
\left|h\left(e^{i(t+\tau)}\right)-h\left(e^{i \tau}\right)\right| & \leq\left|e^{i(t+\tau)}-e^{i \tau}\right|^{1-\alpha}\|h\|_{1-\alpha} \\
& =\left|e^{i t}-1\right|^{1-\alpha}\|h\|_{1-\alpha} \\
& \leq \frac{|t|^{1-\alpha}\left(e^{\pi}-1\right)^{1-\alpha}}{\pi^{1-\alpha}}\|h\|_{1-\alpha} .
\end{aligned}
$$

From (80) and (81), noting that for $0 \leq t \leq \pi$,

$$
\begin{aligned}
\left|e^{i t}-\right| z||^{2} & =(1-|z|)^{2}+4|z| \sin ^{2} \frac{t}{2} \\
& \geq(1-|z|)^{2}+4|z| \frac{t^{2}}{\pi^{2}},
\end{aligned}
$$

we deduce

$$
\begin{aligned}
& \left|h_{z}(z)\right|+\left|h_{\bar{z}}(z)\right| \leq \frac{\left(e^{\pi}-1\right)^{1-\alpha}\|h\|_{1-\alpha}}{\pi^{2-\alpha}} \\
& \cdot \int_{-\pi}^{\pi} \frac{|t|^{1-\alpha}}{\left|e^{i t}-\right| z||^{2}} d t \leq \frac{2\left(e^{\pi}-1\right)^{1-\alpha}\|h\|_{1-\alpha}}{\pi^{2-\alpha}} \\
& \cdot \int_{0}^{\pi} \frac{t^{1-\alpha}}{(1-|z|)^{2}+4|z|\left(t^{2} / \pi^{2}\right)} d t .
\end{aligned}
$$


If $|z| \leq 1 / 2$, then $(1-|z|)^{2} \geq 1 / 4$, so

$$
\begin{aligned}
& \int_{0}^{\pi} \frac{t^{1-\alpha}}{(1-|z|)^{2}+4|z|\left(t^{2} / \pi^{2}\right)} d t \leq \int_{0}^{\pi} \frac{t^{1-\alpha}}{(1-|z|)^{2}} d t \\
& \quad \leq 4 \int_{0}^{\pi} t^{1-\alpha} d t=\frac{4 \pi^{2-\alpha}}{2-\alpha},
\end{aligned}
$$

so from (83), we obtain

$$
\begin{gathered}
\left(1-|z|^{2}\right)^{\alpha}\left(\left|h_{z}(z)\right|+\left|h_{\bar{z}}(z)\right|\right) \\
\leq \frac{8\left(e^{\pi}-1\right)^{1-\alpha}}{2-\alpha}\|h\|_{1-\alpha} .
\end{gathered}
$$

Next assume $1 / 2 \leq|z|<1$. From (83), making the substitution $s=t /(1-|z|)$, and noting that $1>4|z| / \pi^{2}$, we have

$$
\begin{aligned}
& \left|h_{z}(z)\right|+\left|h_{\bar{z}}(z)\right| \leq \frac{2\left(e^{\pi}-1\right)^{1-\alpha}\|h\|_{1-\alpha}}{\pi^{2-\alpha}}(1-|z|)^{-\alpha} \\
& \cdot \int_{0}^{\infty} \frac{s^{1-\alpha}}{1+4|z|\left(s^{2} / \pi^{2}\right)} d s \\
& \quad \leq \frac{2\left(e^{\pi}-1\right)^{1-\alpha}\|h\|_{1-\alpha}}{\pi^{2-\alpha}}(1-|z|)^{-\alpha} \\
& \cdot \int_{0}^{\infty} \frac{s^{1-\alpha}}{\left(4|z| / \pi^{2}\right)\left(1+s^{2}\right)} d s \\
& \quad=\frac{\pi^{\alpha}\left(e^{\pi}-1\right)^{1-\alpha}\|h\|_{1-\alpha}}{2|z|}(1-|z|)^{-\alpha} \int_{0}^{\infty} \frac{s^{1-\alpha}}{1+s^{2}} d s \\
& \quad=C\left(1-|z|^{2}\right)^{-\alpha}\|h\|_{1-\alpha} \\
& \cdot\left(\int_{0}^{1} \frac{s^{1-\alpha}}{1+s^{2}} d s+\int_{1}^{\infty} \frac{s^{1-\alpha}}{1+s^{2}} d s\right) \leq C\left(1-|z|^{2}\right)^{-\alpha} \\
& \cdot\|h\|_{1-\alpha}\left(\frac{\pi}{4}+\int_{1}^{\infty} \frac{s^{1-\alpha}}{1+s^{2}} d s\right),
\end{aligned}
$$

where $C=\pi^{\alpha}\left(e^{\pi}-1\right)^{1-\alpha}$. Noting that

$$
\int_{1}^{\infty} \frac{s^{1-\alpha}}{1+s^{2}} d s \leq \int_{1}^{\infty} \frac{1}{s^{1+\alpha}} d s=\frac{1}{\alpha},
$$

it follows from (86) that for $1 / 2 \leq|z|<1$,

$$
\left(1-|z|^{2}\right)^{\alpha}\left(\left|h_{z}(z)\right|+\left|h_{\bar{z}}(z)\right|\right) \leq C\|h\|_{1-\alpha} .
$$

Therefore, combining the results in the two cases $|z| \leq 1 / 2$ and $1 / 2 \leq|z|<1$, we obtain

$$
\sup _{z \in \mathbb{D}}\left(1-|z|^{2}\right)^{\alpha}\left(\left|h_{z}(z)\right|+\left|h_{\bar{z}}(z)\right|\right) \leq \text { const. }\|h\|_{1-\alpha} .
$$

This proves that $h \in \mathscr{B}_{H}^{\alpha}$ and

$$
\|h\|_{\mathscr{B}_{H}^{\alpha}} \leq \text { const. }\left(|h(0)|+\|h\|_{1-\alpha}\right)=\text { const. }\|h\|_{\operatorname{Lip}_{H}^{1-\alpha}} .
$$

Combining (79) and (90), we obtain the equivalence of the norms $\|\cdot\|_{\mathscr{B}_{H}^{\alpha}}$ and $\|\cdot\|_{\operatorname{Lip}_{H}^{1-\alpha}}$.
We next show that, in analogy to the analytic case, for $\alpha>$ 1 the space $\mathscr{B}_{H}^{\alpha}$ can be identified with the harmonic growth space $\mathscr{A}_{H}^{1-\alpha}$.

Theorem 18. For $\alpha>1$, as sets, $\mathscr{B}_{H}^{\alpha}=\mathscr{A}_{H}^{1-\alpha}$, and the respective norms are equivalent. The little subspaces $\mathscr{B}_{H, 0}^{\alpha}$ and $\mathscr{A}_{H, 0}^{1-\alpha}$ are equal as well.

Proof. First, assume $h \in \mathscr{B}_{H}^{\alpha}$ and let $f, g \in H(\mathbb{D})$ such that $h=f+\bar{g}$ and $g(0)=0$. Then $f, g \in \mathscr{B}^{\alpha}$, so by Proposition 7 in [4], $f$ and $g$ belong to the growth space $\mathscr{A}^{1-\alpha}$. Thus,

$$
\begin{aligned}
\sup _{z \in \mathbb{D}}\left(1-|z|^{2}\right)^{\alpha-1}|h(z)| & \\
\leq & \sup _{z \in \mathbb{D}}\left(1-|z|^{2}\right)^{\alpha-1}|f(z)| \\
& +\sup _{z \in \mathbb{D}}\left(1-|z|^{2}\right)^{\alpha-1}|g(z)|,
\end{aligned}
$$

which is finite. Therefore, $h \in \mathscr{A}_{H}^{1-\alpha}$. The inclusion $\mathscr{B}_{H, 0}^{\alpha} \subseteq$ $\mathscr{A}_{H, 0}^{1-\alpha}$ follows as well.

Moreover, from (91), since the norms in $\mathscr{B}^{\alpha}$ and $\mathscr{A}^{1-\alpha}$ are equivalent, $h(0)=f(0)$ and $g(0)=0$, for some positive constant $C$, we have

$$
\begin{aligned}
\|h\|_{\mathscr{A}_{H}^{1-\alpha}} & \leq\|f\|_{\mathscr{A}^{1-\alpha}}+\|g\|_{\mathscr{A}^{1-\alpha}} \leq C\left(\|f\|_{\mathscr{B}^{\alpha}}+\|g\|_{\mathscr{B}^{\alpha}}\right) \\
& =C\left(|f(0)|+\beta_{f}^{\alpha}+\beta_{g}^{\alpha}\right) \\
& \leq 2 C\left(|h(0)|+\max \left\{\beta_{f}^{\alpha}, \beta_{g}^{\alpha}\right\}\right) \\
& \leq 2 C\left(|h(0)|+\beta_{h}^{\alpha}\right)=2 C\|h\|_{\mathscr{B}_{H}^{\alpha}} .
\end{aligned}
$$

Conversely, suppose $h \in \mathscr{A}_{H}^{1-\alpha}$. Fix $z \in \mathbb{D}$ and set $R=$ $\sqrt{\left(1+|z|^{2}\right) / 2}$. So $1 / \sqrt{2} \leq R<1$. Using (17) and (18), we have

$$
\left|h_{z}(z)\right|+\left|h_{\bar{z}}(z)\right| \leq \frac{R}{\pi} \int_{-\pi}^{\pi} \frac{\left|h\left(R e^{i t}\right)\right|}{\left|R e^{i t}-z\right|^{2}} d t .
$$

Since $h \in \mathscr{A}_{H}^{1-\alpha}$, we have

$$
\left|h\left(R e^{i t}\right)\right| \leq \frac{\|h\|_{\mathscr{A}_{H}^{1-\alpha}}}{\left(1-R^{2}\right)^{\alpha-1}}
$$

Since by our choice of $R, R^{2}=\left(1+|z|^{2}\right) / 2$, we have

$$
1-R^{2}=R^{2}-|z|^{2}=\frac{1-|z|^{2}}{2} .
$$

By these facts and multiplying (93) by $\left(1-|z|^{2}\right)^{\alpha}$, it follows that 


$$
\begin{aligned}
& \left(1-|z|^{2}\right)^{\alpha}\left(\left|h_{z}(z)\right|+\left|h_{\bar{z}}(z)\right|\right) \\
& \leq \frac{R}{\pi} \int_{-\pi}^{\pi} \frac{\|h\|_{\mathscr{A}_{H}^{1-\alpha}}\left(1-R^{2}\right)}{\left|R e^{i t}-z\right|^{2}}\left(\frac{1-|z|^{2}}{1-R^{2}}\right)^{\alpha} d t . \\
& \leq 2^{\alpha}\|h\|_{\mathscr{A}_{H}^{1-\alpha}} \frac{1}{\pi} \int_{-\pi}^{\pi} \frac{R^{2}-|z|^{2}}{\left|R e^{i t}-z\right|^{2}} d t . \\
& =2^{\alpha+1}\|h\|_{\mathscr{A}_{H}^{1-\alpha}},
\end{aligned}
$$

where we used the identity

$$
\frac{1}{2 \pi} \int_{-\pi}^{\pi} \frac{R^{2}-|z|^{2}}{\left|R e^{i t}-z\right|^{2}} d t=1
$$

since the mean of the Poisson kernel for the disk of radius $R$ over the circle of radius $\mathrm{R}$ is 1 . Therefore, $h \in \mathscr{B}_{H}^{\alpha}$.

Finally, since $|h(0)| \leq\|h\|_{\mathscr{A}_{H}^{1-\alpha}}$, from (96) it follows that

$$
\|h\|_{\mathscr{B}_{H}^{\alpha}} \leq\left(1+2^{\alpha+1}\right)\|h\|_{\mathscr{A}_{H}^{1-\alpha}},
$$

which, combined with (92), proves that the norms of $\mathscr{B}_{H}^{\alpha}$ and $\mathscr{A}_{H}^{1-\alpha}$ are equivalent.

Lastly, assume $h \in \mathscr{A}_{H, 0}^{1-\alpha}$. Fix $\varepsilon>0$ and let $r \in(0,1)$ be chosen so that

$$
|h(z)|<\frac{\varepsilon}{\left(1-|z|^{2}\right)^{\alpha-1}}
$$

whenever $r<|z|<1$. Choosing $z$ with $r<|z|<1$ and defining $R$ in terms of $z$ as done previously, from (93) arguing as above, we see that

$$
\begin{aligned}
(1 & \left.-|z|^{2}\right)^{\alpha}\left(\left|h_{z}(z)\right|+\left|h_{\bar{z}}(z)\right|\right) \\
& <\frac{R}{\pi} \int_{-\pi}^{\pi} \frac{\varepsilon\left(1-R^{2}\right)}{\left|R e^{i t}-z\right|^{2}}\left(\frac{1-|z|^{2}}{1-R^{2}}\right)^{\alpha} d t \leq 2^{\alpha+1} \varepsilon .
\end{aligned}
$$

Hence, $h \in \mathscr{B}_{H, 0}^{\alpha}$.

Given $\alpha>0, n \in \mathbb{N}$, and $h$ harmonic on $\mathbb{D}$, let us define

$$
\beta_{h, n}^{\alpha}:=\sup _{z \in \mathbb{D}}\left(1-|z|^{2}\right)^{\alpha+n-1}\left(\left|f^{(n)}(z)\right|+\left|g^{(n)}(z)\right|\right) \text {. }
$$

The following result shows that, in analogy to the analytic case, for any integer $n \geq 2$, the elements of the harmonic $\alpha$-Bloch space and its subspace $\mathscr{B}_{H, 0}^{\alpha}$ can be characterized in terms of the $n^{\text {th }}$ derivatives of $f$ and $g$ and the Bergman weight $z \longmapsto\left(1-|z|^{2}\right)^{\gamma}$, whose exponent $\gamma$ is a certain function of $n$ and $\alpha$.

Theorem 19. Suppose $\alpha>0, n \geq 2$ is an integer, and $h$ is a harmonic mapping on $\mathbb{D}$. Then

(i) $h \in \mathscr{B}_{H}^{\alpha}$ if and only if $\beta_{h, n}^{\alpha}<\infty$.

(ii) $h \in \mathscr{B}_{H, 0}^{\alpha}$ if and only if

$$
\lim _{|z| \rightarrow 1}\left(1-|z|^{2}\right)^{\alpha+n-1}\left(\left|f^{(n)}(z)\right|+\left|g^{(n)}(z)\right|\right)=0 .
$$

Proof. To prove (i), assume $h \in \mathscr{B}_{H}^{\alpha}$. By Theorem 1, there exists $C>0$ such that for each $f$ analytic on $\mathbb{D}, \beta_{f, n}^{\alpha} \leq C \beta_{f}^{\alpha}$.

Let $h \in \mathscr{B}_{H}^{\alpha}$ such that $h=f+\bar{g}$ for some $f, g \in \mathscr{B}^{\alpha}$. Then by Theorem 1 and the inequality (36)

$$
\beta_{h, n}^{\alpha} \leq \beta_{f, n}^{\alpha}+\beta_{g, n}^{\alpha} \leq C\left(\beta_{f}^{\alpha}+\beta_{g}^{\alpha}\right) \leq 2 C \beta_{h}^{\alpha} .
$$

In particular, if $h \in \mathscr{B}_{H}^{\alpha}$, then $\beta_{h, n}^{\alpha}$ is finite.

Conversely, assume $\beta_{h, n}^{\alpha}<\infty$. Then $\beta_{f, n}^{\alpha} \leq \beta_{h, n}^{\alpha}$ and $\beta_{g, n}^{\alpha} \leq \beta_{h, n}^{\alpha}$. Thus, $\beta_{f, n}^{\alpha}$ and $\beta_{g, n}^{\alpha}$ are finite. Therefore, again by Theorem 1, $f, g \in \mathscr{B}^{\alpha}$, and thus by Proposition 10, $h \in \mathscr{B}_{H}^{\alpha}$.

To prove (ii), assume first that $h \in \mathscr{B}_{H, 0}^{\alpha}$. Then $f, g \in \mathscr{B}_{0}^{\alpha}$, so by Theorem 1 ,

$$
\begin{aligned}
& \lim _{|z| \rightarrow 1}\left(1-|z|^{2}\right)^{\alpha+n-1}\left(\left|f^{(n)}(z)\right|+\left|g^{(n)}(z)\right|\right) \\
& \leq \lim _{|z| \rightarrow 1}\left(1-|z|^{2}\right)^{\alpha+n-1}\left|f^{(n)}(z)\right| \\
& \quad+\lim _{|z| \rightarrow 1}\left(1-|z|^{2}\right)^{\alpha+n-1}\left|g^{(n)}(z)\right|=0
\end{aligned}
$$

proving that (102) holds.

Conversely, suppose (102) holds. Then

$$
\begin{aligned}
& \lim _{|z| \rightarrow 1}\left(1-|z|^{2}\right)^{\alpha+n-1}\left|f^{(n)}(z)\right| \\
& \quad \leq \lim _{|z| \rightarrow 1}\left(1-|z|^{2}\right)^{\alpha+n-1}\left(\left|f^{(n)}(z)\right|+\left|g^{(n)}(z)\right|\right) \\
& \quad=0
\end{aligned}
$$

and the same property holds for $g^{(n)}$. Therefore, $f, g \in \mathscr{B}_{0}^{\alpha}$, hence $h \in \mathscr{B}_{H, 0}^{\alpha}$.

\section{Remarks on the Harmonic Growth Spaces}

An immediate consequence of the identification of the harmonic $\alpha$-Bloch space with the harmonic growth space of order $\alpha-1$ is the following result.

Corollary 20. Given $\alpha>0$ and $f, g \in H(\mathbb{D}), f+\bar{g} \epsilon$ $\mathscr{A}_{H}^{-\alpha}$ (respectively, $f+\bar{g} \in \mathscr{A}_{H, 0}^{-\alpha}$ ) if and only if $f, g \in \mathscr{A}^{-\alpha}$ (respectively, $f, g \in \mathscr{A}_{0}^{-\alpha}$ ).

Proof. We only need to prove the necessity. Assume $h:=$ $f+\bar{g} \in \mathscr{A}_{H}^{-\alpha}$. Then by Theorem $18, h \in \mathscr{B}_{H}^{\alpha+1}$. So by Proposition 10, $f, g \in \mathscr{B}^{\alpha+1}$. Therefore, by Proposition 7 of [4], $f, g \in \mathscr{A}^{-\alpha}$. The proof for the little spaces case is similar.

We now apply this result to prove a property of the harmonic growth spaces and their "little" subspaces.

Proposition 21. Let $\alpha>0$ and $\phi, f, g \in H(\mathbb{D})$.

(a) If $\phi$ is bounded and $f+\bar{g} \in \mathscr{A}_{H}^{-\alpha}$ (respectively, $\mathscr{A}_{H, 0}^{-\alpha}$ ), then $\phi f+\overline{\phi g} \in \mathscr{A}_{H}^{-\alpha}$ (respectively, $\mathscr{A}_{H, 0}^{-\alpha}$ ).

(b) If $\phi$ is bounded away from 0 near the unit circle and $\phi f+$ $\overline{\phi g} \in \mathscr{A}_{H}^{-\alpha}\left(\right.$ respectively, $\left.\mathscr{A}_{H, 0}^{-\alpha}\right)$, then $f+\bar{g} \in \mathscr{A}_{H}^{-\alpha}$ (respectively, $\left.\mathscr{A}_{H, 0}^{-\alpha}\right)$. 
Proof. Assume $\phi$ is bounded and $f+\bar{g} \in \mathscr{A}_{H}^{-\alpha}$. Then by Corollary 20, $f, g \in \mathscr{A}^{-\alpha}$, so by the triangle inequality,

$$
\begin{aligned}
& \sup _{z \in \mathbb{D}}\left(1-|z|^{2}\right)^{\alpha}|\phi(z) f(z)+\overline{\phi(z) g(z)}| \leq\|\phi\|_{\infty} \\
& \quad \cdot\left(\sup _{z \in \mathbb{D}}\left(1-|z|^{2}\right)^{\alpha}|f(z)|\right. \\
& \left.\quad+\sup _{z \in \mathbb{D}}\left(1-|z|^{2}\right)^{\alpha}|g(z)|\right)
\end{aligned}
$$

which is finite.

Next, assume $f+\bar{g} \in \mathscr{A}_{H, 0}^{-\alpha}$. Then $f, g \in \mathscr{A}_{0}^{-\alpha}$, so by the triangle inequality and the boundedness of $\phi$,

$$
\begin{aligned}
& \lim _{|z| \longrightarrow 1}\left(1-|z|^{2}\right)^{\alpha}|\phi(z) f(z)+\overline{\phi(z) g(z)}| \leq\|\phi\|_{\infty} \\
& \quad \cdot\left(\lim _{|z| \longrightarrow 1}\left(1-|z|^{2}\right)^{\alpha}|f(z)|\right. \\
& \left.\quad+\lim _{|z| \longrightarrow 1}\left(1-|z|^{2}\right)^{\alpha}|g(z)|\right)=0 .
\end{aligned}
$$

Therefore, $\phi f+\overline{\phi g} \in \mathscr{A}_{H, 0}^{-\alpha}$, proving (a).

Next, assume $\phi f+\overline{\phi g} \in \mathscr{A}_{H}^{-\alpha}$, where $\phi$ is bounded away from zero. Then, again by Corollary 20, the functions $\phi f$ and $\phi g$ are in $\mathscr{A}^{-\alpha}$, and there exist constants $r \in(0,1)$ and $\delta>0$ such that $|\phi(z)|>\delta$ whenever $r<|z|<1$.

We now show that $f, g \in \mathscr{A}^{-\alpha}$. Since $f$ is analytic, it is bounded on each compact subset of $\mathbb{D}$, and thus

$$
\sup _{|z| \leq r}\left(1-|z|^{2}\right)^{\alpha}|f(z)| \leq \max _{|z|=r}|f(z)|<\infty .
$$

On the other hand,

$$
\begin{aligned}
& \sup _{\substack{r<|z|<1\\
}}\left(1-|z|^{2}\right)^{\alpha}|f(z)| \\
& =\sup _{r<|z|<1} \frac{1}{|\phi(z)|}\left(1-|z|^{2}\right)^{\alpha}|\phi(z) f(z)| \\
& \quad \leq \frac{1}{\delta} \sup _{r<|z|<1}\left(1-|z|^{2}\right)^{\alpha}|\phi(z) f(z)|<\infty .
\end{aligned}
$$

Therefore, combining (108) and (109), we have

$$
\begin{aligned}
\sup _{z \in \mathbb{D}}\left(1-|z|^{2}\right)^{\alpha}|f(z)| \\
\leq \sup _{|z| \leq r}\left(1-|z|^{2}\right)^{\alpha}|f(z)| \\
\quad+\sup _{r<|z|<1}\left(1-|z|^{2}\right)^{\alpha}|f(z)|<\infty,
\end{aligned}
$$

proving that $f \in \mathscr{A}^{-\alpha}$. The same argument shows that $g \in$ $\mathscr{A}^{-\alpha}$. Therefore, by Corollary $20, f+\bar{g} \in \mathscr{A}_{H}^{-\alpha}$.

Next, assume $\phi f+\overline{\phi g} \in \mathscr{A}_{H, 0}^{-\alpha}$. Then, by Corollary 20, the functions $\phi f$ and $\phi g$ are in $\mathscr{A}_{0}^{-\alpha}$. Fix $\varepsilon>0$ and choose $r \in(0,1)$ and $\delta>0$ such that $|\phi(z)|>\delta$ and $(1-$ $\left.|z|^{2}\right)^{\alpha}|\phi(z) f(z)|<\varepsilon$ whenever $r<|z|<1$. Then, for $r<|z|<1$,

$$
\left(1-|z|^{2}\right)^{\alpha}|f(z)| \leq \frac{1}{\delta}\left(1-|z|^{2}\right)^{\alpha}|\phi(z) f(z)|<\frac{\varepsilon}{\delta},
$$

proving that $f \in \mathscr{A}_{0}^{-\alpha}$. Arguing similarly, we see that $g \in$ $\mathscr{A}_{0}^{-\alpha}$. Therefore, again by Corollary 20 , we have $f+\bar{g} \in \mathscr{A}_{H, 0}^{-\alpha}$, completing the proof of (b).

The following result is an immediate consequence of Proposition 21.

Corollary 22. Let $\alpha>0, f, g \in H(\mathbb{D})$, and $p$ a polynomial in $z$ with no zeros on the unit circle. Then, $f+\bar{g} \in \mathscr{A}_{H}^{-\alpha}$ (respectively, $\mathscr{A}_{H, 0}^{-\alpha}$ ) if and only if $p f+\overline{p g} \in \mathscr{A}_{H}^{-\alpha}$ (respectively, $\left.\mathscr{A}_{H, 0}^{-\alpha}\right)$.

The results of Proposition 21 and its corollary do not hold if $\phi$ is unbounded or $\phi(z)$ is not bounded away from 0 as $z$ approaches the unit circle. For example, if $\phi(z)=f(z)=$ $g(z)=(1-z)^{-\alpha}$, then

$$
f(z)+\overline{g(z)}=2 \operatorname{Re}\left[(1-z)^{-\alpha}\right],
$$

which is in $\mathscr{A}_{H}^{-\alpha}$, but $\phi f+\overline{\phi g} \notin \mathscr{A}_{H}^{-\alpha}$. On the other hand, if we choose $\phi(z)=(1-z)^{\alpha}$ and $f(z)=g(z)=(1-z)^{-2 \alpha}$, then $\phi f+\overline{\phi g} \in \mathscr{A}_{H}^{-\alpha}$ while, $f+\bar{g} \notin \mathscr{A}_{H}^{-\alpha}$.

\section{Data Availability}

Data is not needed since this research does not involve data.

\section{Conflicts of Interest}

The authors declare that they have no conflicts of interest.

\section{References}

[1] F. Colonna, "Bloch and normal functions and their relation," Rendiconti del Circolo Matematico di Palermo, Serie II, vol. 38, no. 2, pp. 161-180, 1989.

[2] K. Zhu, Operator Theory in Function Spaces, Mathematical Surveys and Monographs, American Mathematical Society, Providence, RI, USA, 2nd edition, 2007.

[3] F. Colonna, "The Bloch constant of bounded harmonic mappings," Indiana University Mathematics Journal, vol. 38, no. 4, pp. 829-840, 1989.

[4] K. H. Zhu, "Bloch type spaces of analytic functions," Rocky Mountain Journal of Mathematics, vol. 23, no. 3, pp. 1143-1177, 1993.

[5] R. Yoneda, "A characterization of the harmonic Bloch space and the harmonic Besov spaces by an oscillation," Proceedings of the Edinburgh Mathematical Society, vol. 45, no. 1, pp. 229-239, 2002.

[6] S. H. Chen, S. Ponnusamy, and X. Wang, "Landau's theorem and Marden constant for harmonic $v$-Bloch mappings," Bulletin of the Australian Mathematical Society, vol. 84, no. 1, pp. 19-32, 2011. 
[7] S. Chen, S. Ponnusamy, and X. Wang, "On planar harmonic Lipschitz and planar harmonic Hardy classes," Annales- Academiae Scientiarum Fennicae Mathematica, vol. 36, no. 2, pp. 567-576, 2011.

[8] S. Chen, S. Ponnusamy, and A. Rasila, "Lengths, areas and Lipschitz-type spaces of planar harmonic mappings," Nonlinear Analysis. Theory, Methods \& Applications. An International Multidisciplinary Journal, vol. 115, pp. 62-70, 2015.

[9] S. Chen and X. Wang, "On harmonic Bloch spaces in the unit

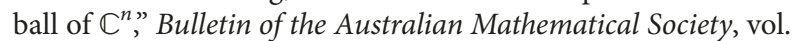
84, no. 1, pp. 67-78, 2011.

[10] K. Stroethoff, "Harmonic Bergman spaces," in Holomorphic spaces, vol. 33 of Math. Sci. Res. Inst. Publ., pp. 51-63, Cambridge Univ. Press, Cambridge, 1998.

[11] S. Chen, S. Ponnusamy, and X. Wang, "Harmonic mappings in Bergman spaces," Monatshefte für Mathematik, vol. 170, no. 3-4, pp. 325-342, 2013.

[12] X. Fu and X. Liu, "On characterizations of Bloch spaces and Besov spaces of pluriharmonic mappings," Journal of Inequalities and Applications, vol. 2015, article 360, 10 pages, 2015.

[13] P. Duren, Harmonic Mappings in the Plane, Cambridge University Press, London, UK, 2004.

[14] M. Aljuaid and F. Colonna, "On the harmonic Zygmund spaces," In press.

[15] H. Hedenmalm, B. Korenblum, and K. Zhu, Theory of Bergman Spaces, Springer-Verlag, New York, NY, USA, 2000.

[16] J. B. Conway, Functions of One Complex Variable I, SpringerVerlag, New York, NY, USA, 2nd edition, 2001.

[17] F. Colonna and M. Tjani, "Operator norms and essential norms of weighted composition operators between Banach spaces of analytic functions," Journal of Mathematical Analysis and Applications, vol. 434, no. 1, pp. 93-124, 2016. 


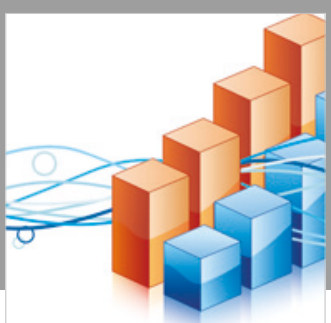

Advances in

Operations Research

\section{-n-m}
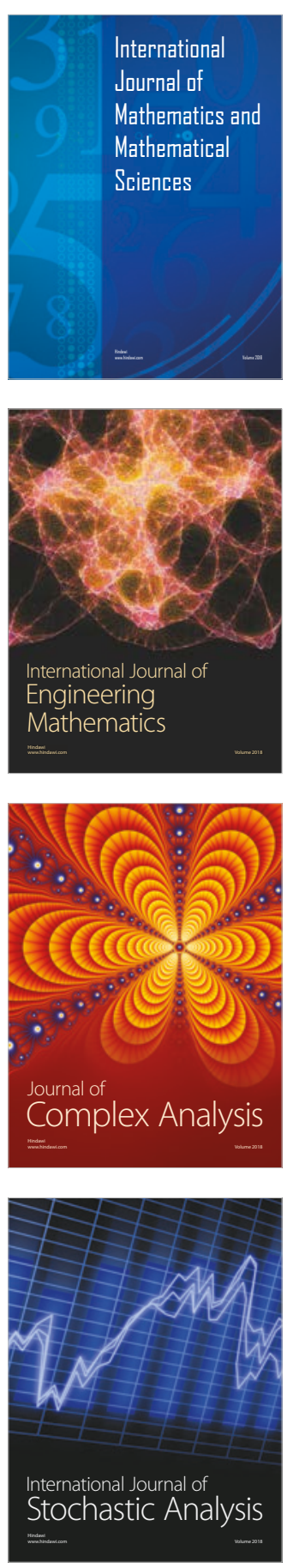
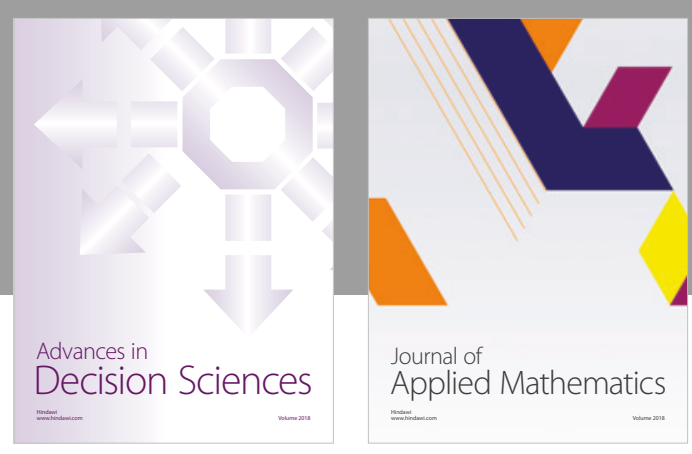

Journal of

Applied Mathematics
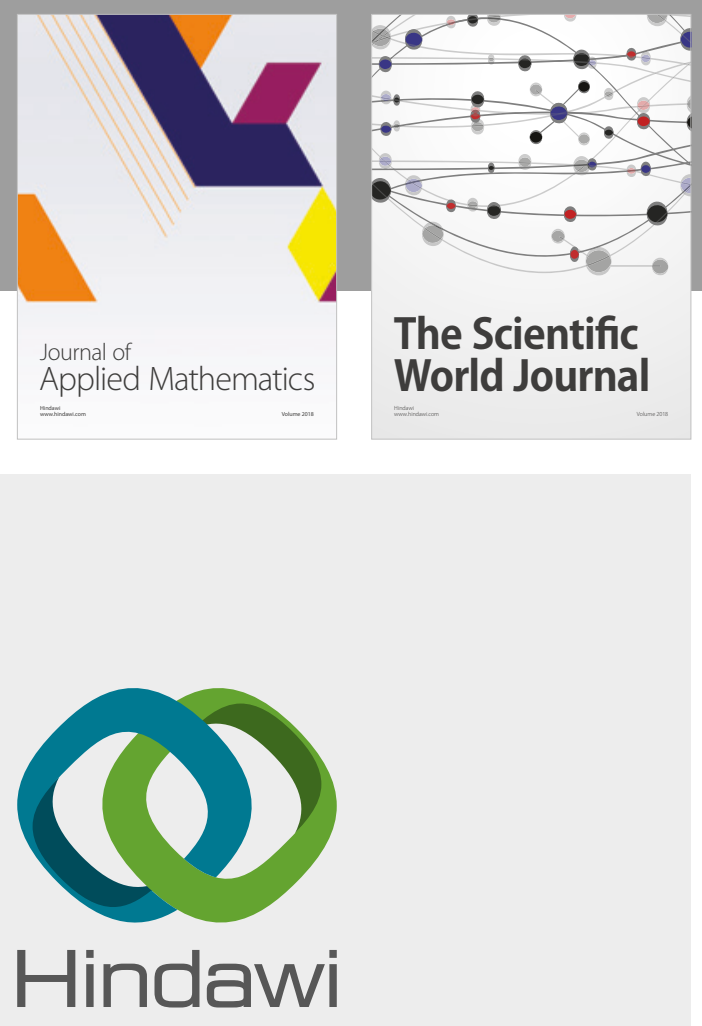

Submit your manuscripts at

www.hindawi.com

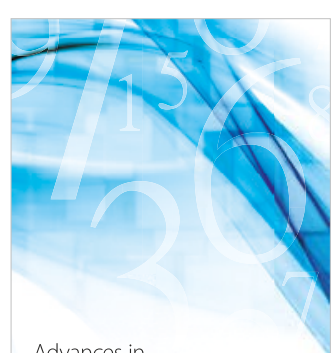

Advances in
Numerical Analysis
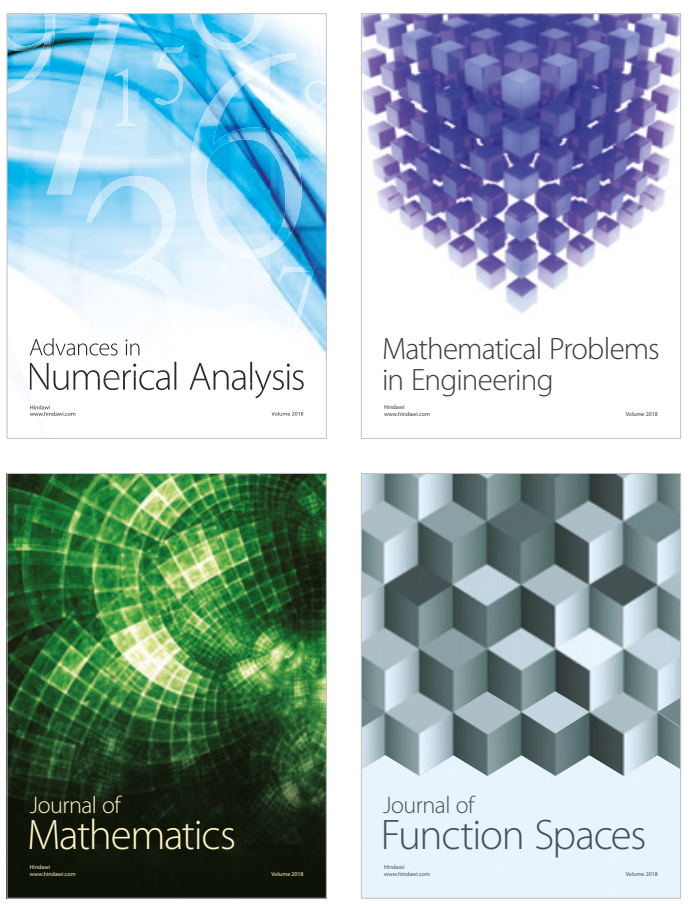

Mathematical Problems in Engineering

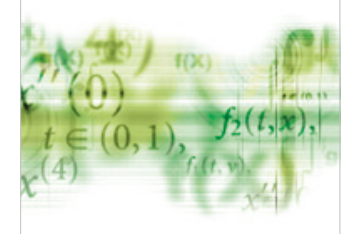

International Journal of

Differential Equations

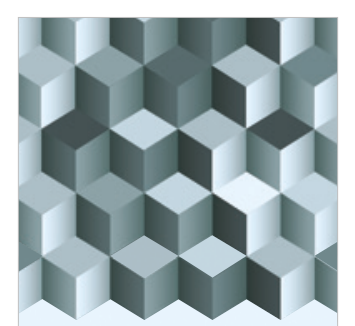

Journal of

Function Spaces

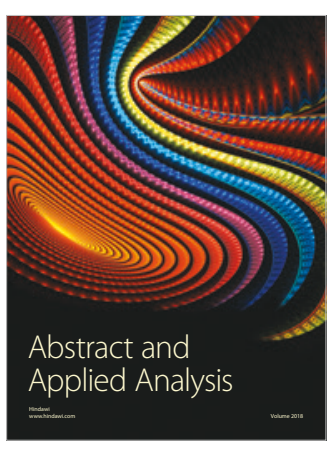

The Scientific

World Journal

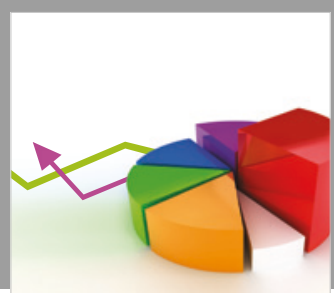

Journal of

Probability and Statistics
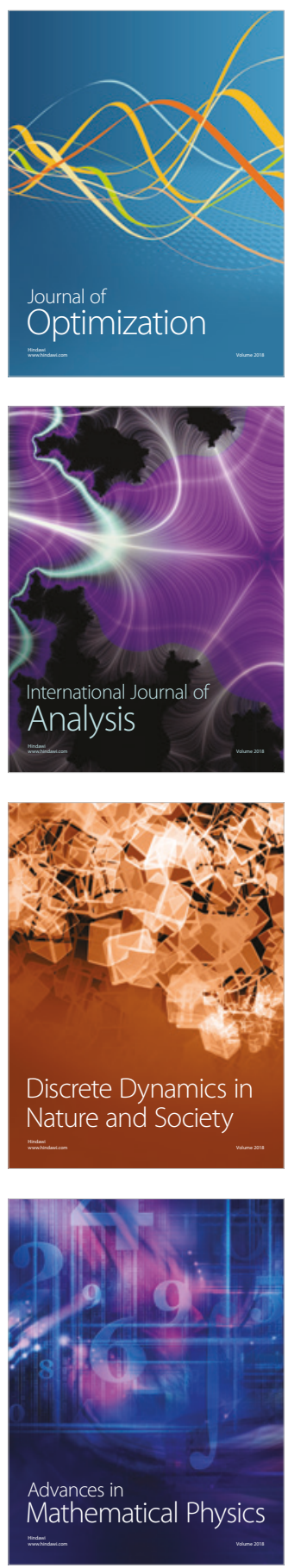